\title{
DRAWING AND CONCEIVING SPACE: HOW TO EXPRESS SPATIAL EXPERIENCE THROUGH DRAWING?
}

\section{Robin Schaeverbeke, Hélène Aarts, Ann Heylighen,}

\begin{abstract}
Teaching drawing in architectural education raises questions regarding the representation of spatial experiences: to what extent can sensory experiences of space be intensified through observing and drawing and, perhaps equally important, what those drawings would look like?

In the context of their drawing classes, the authors started to inquire the discrepancy between conceiving and perceiving space, and the aptitude of representing spatial concepts upon a two dimensional surface. Through observation and translating observation into drawings, students discover that conventionalised ways of drawing, such as linear perspective, only reveal part of the story. While linear perspective remains the dominant way of representing space, obviously visible in photography, film, 3D-imaging and architectural impressions, the authors started looking for ways of drawing which inquire possibilities of expressing spatial experiences. Drawing as an activity which is able to enhance spatial understanding, rather than as a tool to communicate virtual spaces. Next to drawing as a 'skill', which can be learnt, the drawing classes started to inquire non-visual aspects of space by analysing attributes of spatiality, which are difficult to convey through two dimensional drawings.

Starting from a contextualisation of spatial drawing within architectural practice, the article examines the discrepancy between geometric space and lived space, in order to reveal the dubious role of linear perspective within (architectural) culture and history. After a brief return to how we imagined and represented space in our childhood, the article presents a series of practice based examples. Drawing on the authors' teaching practice, it illustrates possibilities to expand our visual language by exploring space and spatiality through observing and drawing.
\end{abstract}

Keywords: Architecture, Drawing, Pedagogy, Representation, Spatial Experience.

\section{L'ESSENTIEL EST INVISIBLE POUR LES YEUX}

In Antoine de Saint Exupéry's famous book 'Le Petit Prince' (1943) the protagonist reveals his simple little secret: 'Il est très simple, on ne voit bien qu' avec le cœur. L'essentiel est invisible pour les yeux.'. To illustrate this statement, the protagonist tells the story in which the Little Prince asks him to draw a sheep. Without hesitation, the protagonist takes out a little piece of paper and draws a sheep. The Prince looks attentively but says: 'This one looks already ill, draw me another one'. After a second, and even a third attempt are rejected, the protagonist - losing his patience - draws a box with three air holes and says: "The sheep you want resides in the box". The Little Prince suddenly looks satisfied: 'That's precisely how I wanted it.' The vented box in de Saint Exupéry's story could be seen as metaphor for spatial representation in architecture. Architects constantly face the incongruence between the embodied experience of space and how to translate that experience into a drawing.

Architectural drawing pedagogy is traditionally directed towards a geometric understanding of our surroundings. Basic lines and planes are considered as a stepping stone to introduce projective drawing and linear perspective. While these tools communicate architectural ideas, we postulate that they reveal only part of the story. As architect and theoretician Juhani
Pallasmaa (2005) emphasises, spatial experiences surpass the visual, they are personal, dynamic and involve all of our senses. If spatial experience involves all our senses, what kind of drawing allows to convey these sensory qualities of space? Which paths can we possibly explore in order to enhance spatial knowledge? To what extent can drawing help to intensify such a spatial experience, and what kind of drawing allows us to express it? What kind of conditions can we create to incorporate the experience of space within drawing classes?

\section{HOW TO CONCEIVE SPACE?}

"A thermometer indicates the temperature visually by the length of the mercury column, but it does not give us the experience of warm and cold" (Arnheim R. $1954,228)$.

Since architects usually do not construct their own buildings, a design, until it is completed, only exists in the minds of the people involved in the design and building process, and in the representational artifacts they use. (Vermeersch P.W. 2013; Medway P. 1996). Representation here, as perceptual psychologist Rudolf Arnheim (1954) points out, does not produce a replica of the object, but its structural equivalent in a given medium. All the knowledge about the virtual 
building is stored in representational artefacts, but also carried in people's situated knowledge, words and gestures (Vermeersch P.W. 2013). Precisely this virtual aspect may be worrying when teaching architectural students 'how to draw', because an all too radical exclusion of the experiential, when imagining virtual spaces, runs the risk of producing empty boxes; boxes which lack perceptual and sensory information marking our spatial experiences.

The introduction of geometry has enabled us to represent the world as a quantifiable phenomenon, characterised by the point, line, curve, surface and figure (Hernandez E. 2013). Science considers space as a homogeneous medium, where things are identified according to their three dimensions. But, as philosopher Maurice Merleau Ponty (1948-2002) remarks, the idea that object characteristics remain constant, denies our ability to mentally change the identity of an object by merely displacing it. Scientifically speaking, the displacement does not alter the object's geometric features, only its physical conditions change. The theoretical concept of pure space, which science has put forward, sharply contrasts with the spectacle brought to us by our senses. With this contrast, Merleau Ponty draws our attention to the fact that the scientific idea of pure space can exist only as a hypothetic concept, because we are quite unable to relate to space in such a detached way, as a kind of body-less object. We, Merleau Ponty continues, are all part of that space, we are one with it. Looking for a definition of space, author Eckhart Tolle (1997) juxtaposes the concept of space with the concept of silence: "If there were nothing but silence, silence would not exist; it is only when we hear sounds that silence is able to surface". Thus, Tolle continues, space cannot exist if there were nothing but space and no objects in it. Nothing can exist without space, while space, in essence, is nothing: space is "not - something" (Tolle E. 1997). In other words, what we regard as sensory qualities are, a priori, not there. They can only exist through our personal experience and relation to space. The whole concept of pure space, as architecture historian and theoretician Jacob Voorthuis (2012) states, is essentially inaccessible to us. We can only know the space which we are able to experience through our own body. Voorthuis considers physical space as the world of tactile matter, of form, colour, light and texture. Pure space is space even before it acquires meaning through our perception: it is not space as we experience, but space as it is outside of us.

Our everyday perception of space is not quantitative but qualitative. Our experience of space is pre-mathematical - it precedes our mathematical understanding. Phenomenology has taught us that the experience of space is characterised not by points but by place, not by measurement but by orientation. Our ability to look about on all sides, examining all the circumstances that may affect the experience, is what characterises the difference between geometric and lived space. This kind of (subjective) spatiality is such that we do not start from where our body is but, rather, we begin over there, where our senses are dwelling at any given moment. We do not perceive pure space, a pure up and down or behind, instead we discover and interpret positions and distances on the paths and ways of everyday associations, which are not ascertained and catalogued by the conventional measurement of space (Hernandez E. 2013).

Taking these sensory issues into account poses severe questions for anyone involved in the pedagogy of perceiving, conceiving and drawing space. Any attempt to visualise spatial experiences is bound to leave the quantifiable framework of geometry to enter the uncertainty of the experiential. As Voorthuis (2012) points out, our ideas about space are influenced by our individual and unique perception of space. As such space becomes more than the surfacing and positioning of forms and materials in some kind of abstract void. Our preconception about the world's functioning and behaviour, combined with the way our body experiences situations, shapes our perception of the world. We do not live in a space that is made up of objectively organized things, we live in a world that concerns us (Hernandez E. 2013). As Arnheim's thermometer illustrates, the feeling of warm and cold surpasses the metric position of the mercury column. Colours, lighting, materiality, sound, personal mood and history, among other things, all shape our experience of warm and cold.

\section{THE SHADOW OF THE MIRROR}

The optical and geometric proof that projective drawing, linear perspective and their family members allow to accurately describe the world, enabled $15^{\text {th }}$ century artists to establish drawing, as 'disegno', into academia (Hill J. 2005). To this end, drawing was stripped of its subjectivity to resurface as a standardised, mathematical tool for visualising and even measuring form and space. The architectural intelligence of the Renaissance was quick to appropriate the newly found geometric tools of imagination to establish them as the lingua franca of architectural and building practice.

The idea of realism was the late and laboriously accomplished product of such sophisticated cultures as Hellenism and the Renaissance. Its evolution can be traced in workshop ploys, which were refined and improved over generations of painters better to suggest pictorial depth. These ploys used geometric elements, but they were used in an intuitive, piecemeal way (Evans R. 2000). Since its introduction, linear perspective gradually became the dominant way of communicating visual information. The tendency to present linear perspective as a superior tool led sociologist and philosopher Bruno Latour (1986) to draw a link between how cultures make the world visible and how a culture understands that world. Because perspective drawing appeared as such a powerful tool, it was adopted in the scientific world and additionally influenced science to go in a direction that further developed technologies of optical consistency: lenses, mirrors, microscopes, ultrasound images, etc. Architectural design followed a similar path, exploring projective and perspective drawings, and later photography and photorealistic rendering technology, to analyse existing places and design and conceive new ones.

Since its introduction, the principle of linear perspective has cast a dark shadow upon our imagination. This shadow severely obscured other ways of imagining the world and eventually we were led to uncritically interpret frozen mirror images as a certified (mirror) view of the world out there. By doing so, we tend to ignore the full density and richness of day-today spatial experiences and how to record them. 


\section{BACK TO THE PLAYGROUND}

In searching for experiential ways of drawing, we could perhaps look back to our childhood and rediscover some of our 'pre-mathematical' attempts to capture the world around us. During our childhood, the world had many faces and when we attempted to draw that world, we drew its physiognomy, its general form or appearance. Children draw what they experience and this experience is related to what the child assimilates emotionally, affectively and functionally. Children constantly re-contextualise the things they have seen and these experiences are constantly transformed by new experiences. So, when children ignore certain details in their expressions, this is only because those details do not play a significant role in the experience (Van Dongen, N., Vanengbersen, E., Meeuwis, W., Mous, M.,s.d.). Rudolf Arnheim (1954, 159) acknowledges the experiential character of children's drawings stating that "children draw what they see, but they see more than they draw". According to Arnheim our youthful perception is made up by the formation of perceptual concepts, our grasping of integral features of a structure. Children generally start to draw from bodily movement, Arnheim continues. Their motor control is not complete yet, but their lines indicate what the drawing is supposed to be like. The imprecision of their strokes gives way to an exactness that is more than sufficient to show what the child is trying to do. "If one wants to represent the roundness of the head, one cannot use the shapes actually given in the head, but one must find or invent a shape that will embody the visual generality of 'roundness' in the world of tangible things". Only after laborious experimentation, children come to draw the head of human being as a circle. For Arnheim this represents a genvine invention (Arnheim R. 1954).

Only gradually children discover and accept the fact that a visual object on paper can stand for an enormously different one in nature. "It takes a great deal of spoiling before we come to think that representation is not only an imitation of the object, but also of its medium, so that we expect a painting not to look like a painting but like a physical space, and a statue not like a statue but like a living body of flesh and blood" (Arnheim R. 1954,163). In returning to our childhood, we also return to our unspoilt view upon the world and, perhaps more importantly, a certain freedom in representing our observations.

\section{STEPPING OUT OF THE SHADOW, DRAWING SPACE}

In order to open ourselves to space through all of our senses we have to immerse ourselves in real time space. Conscious spatial experiences draw attention to the visual, as well as the non-visual aspects of space. Both, visual and non-visual information, should find a place in our graphic explorations, because they mutually shape our understanding of space. Regarding our senses as equally important tends to redefine the drawing classes. Rather than a place to learn preconceived skills, the classes become a laboratory to study the translation of sensory and physical, spatial experiences. Within the laboratory, both the significance as well as the language to signify an observation is inquired. As a result, the teacher has to accept to deviate from preconceived visual conven-

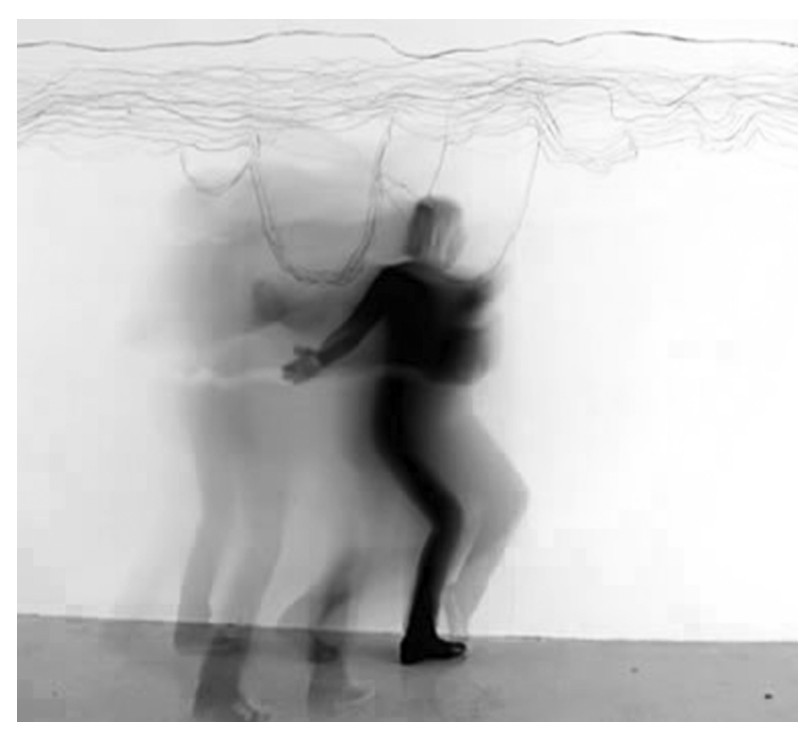

Figure 1. Artist Jane Grisewood combining performance and drawing as a way to connect with space and time (Grisewood, 2013).

tions and the student has to decondition her/himself of any preconceptions regarding visualising perception. Both the teacher and the student are required to leave their pictorial comfort zone by converting drawing to multiple points of view informed by the senses.

In what follows, we will illustrate some practice-based examples of drawing exercises, which aim to extend the language of drawing through addressing other senses than the visual: (i) positioning the drawing body in space as a way to connect a physical experience to the surrounding space; (ii) drawing in the dark by blinding the visual realm and allowing other sensory stimuli to influence the observation and the resulting drawing; (iii) specific drawing techniques, such as wrapping and negative space as an attempt to convert hand and eyes to perform other sensory functions; (iv) adding or isolating other senses to confront the draughtsman with non-visual ways of representing experiences; and finally (v) drawing from (spatial) memory as drawing from the ability to recollect the specificity of (a) spatial experience(s).

(i) Bodily contact, exploring real-time space, being able to move around and dwell in a space, opens up our awareness of the dynamics of spatial experiences. There are several ways to analyse space starting from the human body as a point of reference for instance:, by using the body as a marker where one's movements leave marks upon walls and floors. Marking space through movement reveals features of a space based upon occupation rather than interpretation. Another possibility is analysing movement. Movement reveals information about the various ways in which a body is able to move about in a space, revealing relationships between different elements making up that space. How 'large' or 'tiny' a body is in relation to a space is analysed as the resultant of position, movement and the room's scale and properties (McCall, C. and Grisewood, J. 2011). Drawing while moving (Figure 1) is another way to step away from frozen perspectives. According to artists Mick Maslen and Jack Southern (2011) drawing while moving relinks our eye and hand synchronicity. When observing movement, the draughtsman is confronted with a continuous shift- 

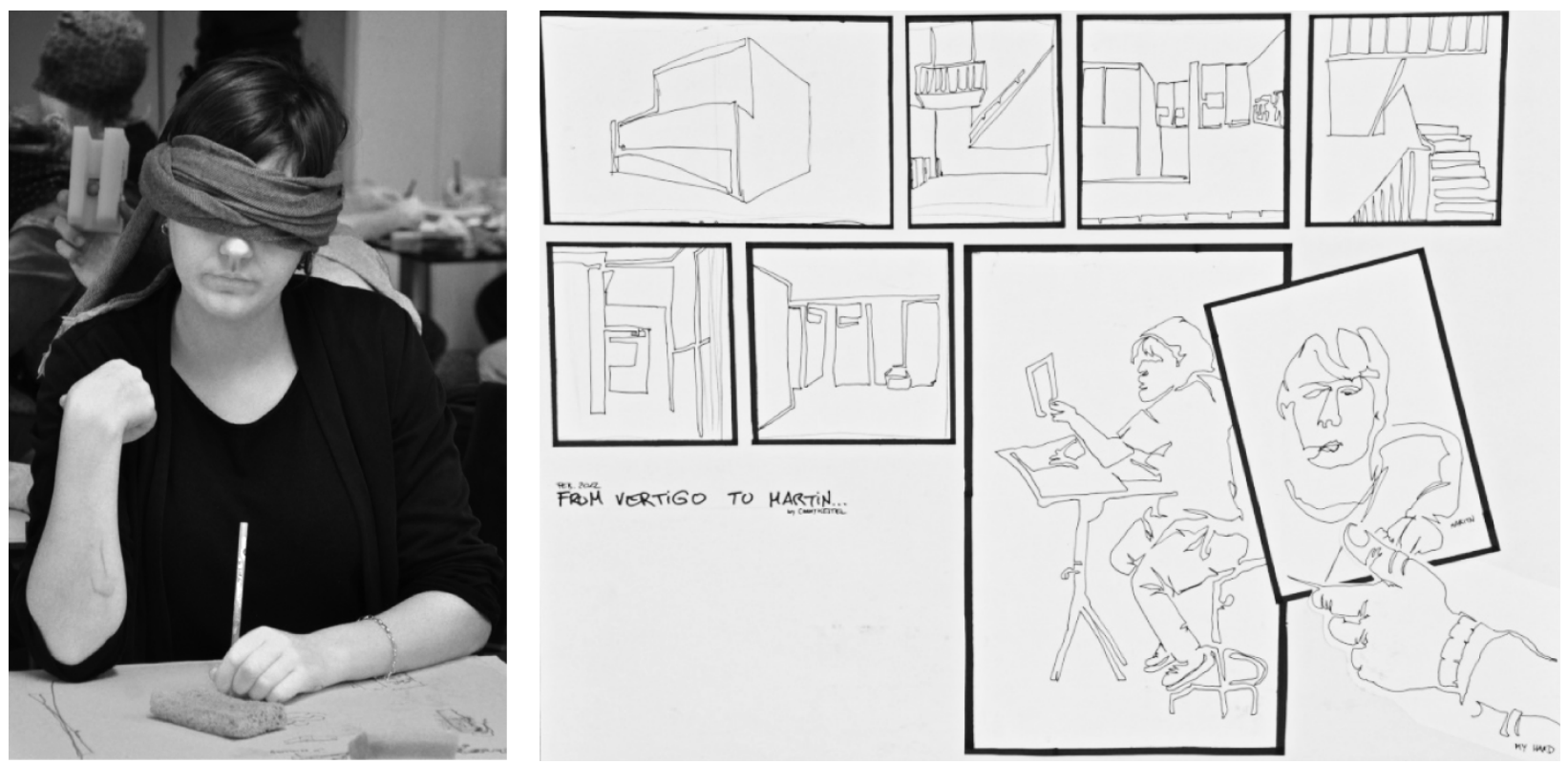

Figure 2. Drawing in the dark blindfolded left) or without looking at the drawing (right) (images by the authors).

ing of focus, because moving bodies do not stand still. Drawing from movement questions preconception and forces the draughtsman to inquire other ways of mapping experience. Mapping techniques, musical or dance notation, diagrams, tracings suddenly become course material and reveal possible routes to represent the experience.

(ii) Drawing in the dark, by touch or without looking at the drawing paper, confronts the draughtsman with the dominance of the visual. Unable to rely upon known drawing strategies, this exercise forces the draughtsman to translate experience into images, without interference or qualification of the visual drawing during the process. As such, the draughtsman is not hampered by her/his preoccupation about how a drawing should look. In its most extreme form, the eyes are excluded from the experience, for instance, through drawing things which are hidden in non-transparent bags. One hand touches the object while the other hand draws (Figure 2). Moulding a form of clay under the table while simultaneously drawing the mould without looking at it introduces haptics into the drawing process. The pencil moves simultaneously with the touching. According to Maslen and Southern (2011), drawing blind creates a direct route between the two hands. It appears that touching without seeing draws our attention to other characteristics than visual observation. In their drawings our students revealed that the crown of a pineapple, when drawn from touch, appeared as more important and larger than when drawn from visual perception alone.

Figure 2: drawing in the dark: blindfolded (left) and without looking at the drawing (right) (images by the authors)

Another way of cutting off the eyes from the observation is drawing without looking at the drawing itself. The idea is that eyes perform the act of touching. As such the eyes move around space and the movement of the eyes and those of the drawing hand are coordinated. The inability of looking at the drawing disengages the mechanism of controlling the expression while drawing. Not looking at the drawing confronts the draughtsman with her/his delusion of expecting the drawing to conform to some sort of preconceived aesthetic scheme. When drawing blind there is no preconceived order, the observing eye leads the drawing hand. The activity enables the draughtsman to switch between the object and the space around it, moving from one space to another. One student testified that after a while her hand stopped drawing immediately when she stopped observing.

(iii) Wrapping, or cross contour drawing (Nicholaides K. 1990, Cooper D. 2007) is another way of allowing touch to direct the drawing (Figure 3). Suggesting the third dimension through using a continuous line which is virtually wrapped around the object one is holding as if strings of rope are spun around it. Again an immediate relation between the observer and the object informs the draughtsman about the object's spatial constellation.

Negative space is another way to search for the space surrounding an object. Negative space is an established exercise within art drawing courses. It is often linked to a Buddhist's awareness for the non-material and so-called negative (Maslen M. and Southern J. 2011 , Edwards B. 1989). Negative space establishes a relation between a form and its background. The change of focus forces the draughtsman to study the interaction between form and space, between subject
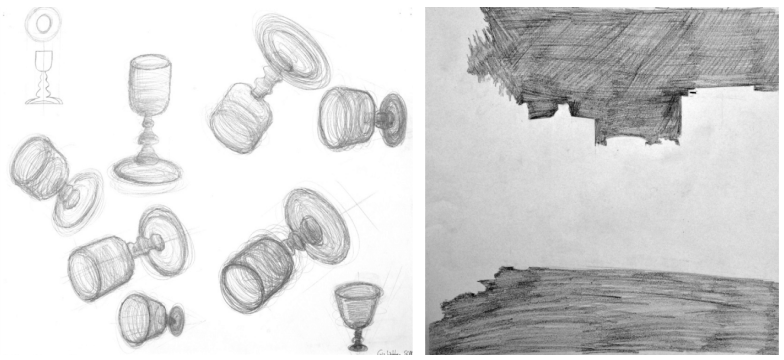

Figure 3. Wrapping (left) and negative space (right) (images by the authors). 
and its surroundings. Within this exercise an object is studied not by drawing the thing in itself, but by building its contours up from the surrounding space. This exercise avoids our inclination to start drawing from lines with the lines functioning as a boundary between objects and the space around them. Wrapping or drawing negative space breaks with one's perceptual habits.

(iv) Other senses than touch and vision also offer invaluable spatial information. Hearing, smell and taste inform our spatial awareness. Music, spices and flavours confront the draughtsman with yet another difficulty. How to translate non visual concepts into a drawing or expression? Memories of sounds, smells and tastes are deeply rooted in our bodily experience of the world. Drawing from these other senses draws attention to the link between all of our senses. A possible exercise to connect with auditory stimuli is translating an experienced musical composition into colour and form (Figure 4). Colour can be used as a tool to describe a composition in terms of rhythm, harmony and mood. When the musical experience is repeated a blinded draughtsman is invited to trace the movement of the music, using two colours only, one in each hand. To conclude the exercise, a graphical score is produced to summarise the musical experience. As such the music challenges one's ability to translate sound into colour by juxtaposing and balancing two colours by movement. This exercise reveals a kind of uniformity in the imagery the draughtsman use to translate sound into form. The resulting forms, colours, movements and composition reveal striking resemblances. Variations on this exercise can be explored by using tastes, smells and other non-formal elements as a driving force for the drawings. (v) Another exercise draws from our memory and storytelling abilities as a means to (re)discover spaces. We all have memories of spatial experiences. Those memories are cerebral and manifest themselves as a kind of collage of impressions (Van der Linden, 2013). They surpass the visual, because our recollections are both made up and distorted by a combination of the sensory experience and our interpretation of the experience. Sofie Van der Linden's work expresses her ability to record the remembrance of spatial experiences. Visiting people in their houses, talking to them, making small sketches of spaces and details as a memory aid, trying to capture the atmosphere of a space and its inhabitant, Van der Linden composes a mental map of the spaces she visits. In her studio she meticulously tries to reconfigure her observation in large scale drawings. Her choice of perspective mode varies, and can best be described by a non-mathematical parallel perspective. But she also allows plan views, projections and mixed forms. Layers of information sometimes overlap, they sometimes contradict each other and certain spots are deliberately left empty. They represent the blank spots in our mind (Van der Linden, 2013). Sometimes a story directs her drawing, sometimes she is the director of her own story, her own recollection of the space she personally experienced. These kinds of drawings make the subjectivity of spatial encounters explicit. A cup of coffee, an abandoned book, unmade beds, visualise recognisable traces of life and reveal possible stories for us to imagine. Here again the human factor is more than a silhovette inhabiting space. The silhovette actually changes the space, defines it. In trying to incorporate the traces of life, the imagined spaces become more vivid.
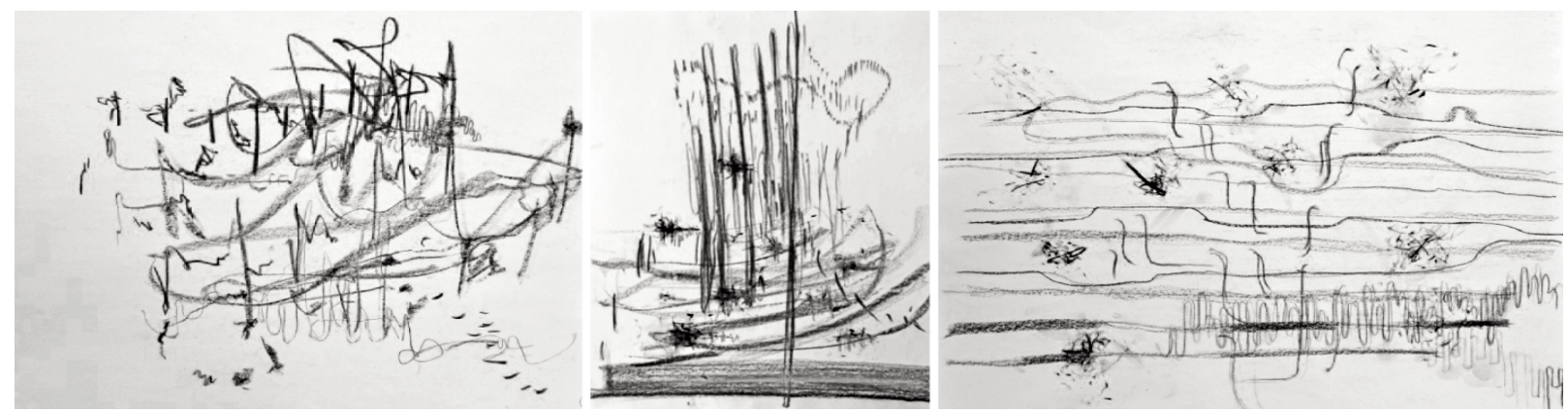

Figure 4. Translating sound (images by the authors).
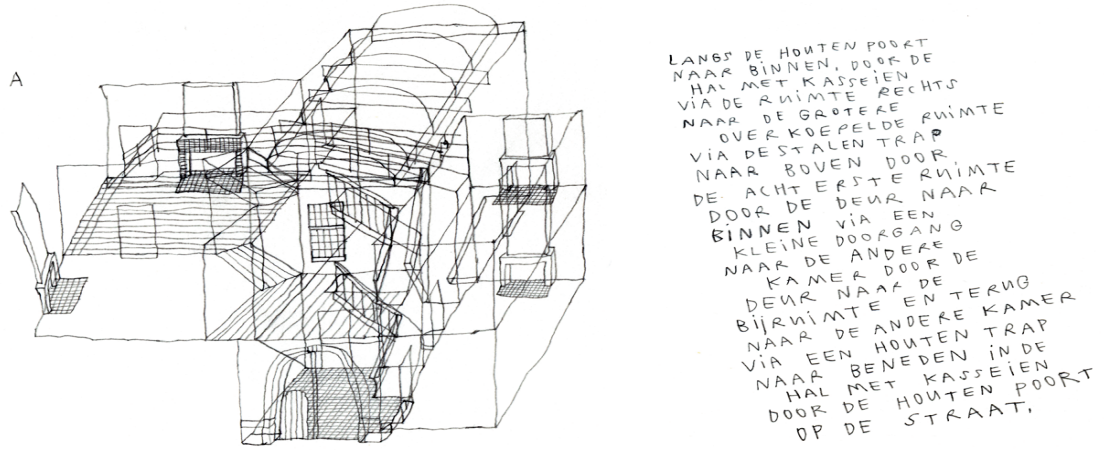

Figure 5. Sofie Van Der Linden: Schippershof (Drawing and Text) (image courtesy of the artist). 


\section{CONCLUSION}

The exercises illustrated above confront the draughtsman with the tendency to over evaluate visual stimuli when observing space. We are inclined to see what we think to see and to relate what we think to see to things we already know. In order to study ways of imagining space upon a two dimensional plane, the exercises invite students to look beyond the conventional boundaries of projection and linear perspective. These kinds of exercises, in our view, should complement conventionalised drawing, in order to raise awareness about the relative character of the convention by drawing attention to multivariate points of reference. In inquiring the imagination of space, cultural, historical, technical, mathematical as well as sensory information should complement each other, in order to explore the full scope of experiencing space. As such we try to expand the draughtsman's knowledge and vocabulary upon spatial matter, in order to enable her/him to choose a personal way of expression, corresponding to the intended imagination.

Opening up the drawing classes to the sensory aspects of spatial experiences, diverts attention from the known to the interpretative, towards personal experience. Through exploring concepts to represent the sensory phenomena, the draughtsman is confronted with perceptual layers which make up space and with the limitations of the medium of drawing, to express certain of these phenomena. Opening up the drawing classes to the sensory means, we have to inquire possibilities to represent experiences, both by provoking thought and by practically illustrating how a drawing, a tool or a technique works. In order to step out of the mirror's shadow, we have to explore graphic possibilities to convey the experience of space, by and through drawing.

In order to enhance spatial awareness, we believe conventional drawing should be complemented by non-mathematical expressions of space. Designing spaces involves studying what it is (a) space is made of. Architectural space is something corporeal and the human body, as an inhabitant of space, has to find its place within it. Ignoring the sensory runs the risk of reducing architectural imagination to a detached concept of non-embodied space.

\section{REFERENCES:}

ARNHEIM, R. 1954, Art and Visual perception, London: Faber and Faber

COOPER, D., 2007, Drawing and Perceiving, Real World Drawing For Students of Architecture and Design, United States, John Wiley and Sons Inc.

DE Saint EXUPERIE, A. 1943, Le Petit Prince, New York, Reynal and Hitchcock

EDWARDS B, 1989, Drawing on the right side of the brain, New York, G.P.Putnam's Sons

EVANS, R., 2000, The Projective Cast, Massachusetts, MIT Press

HERNANDEZ, H., 2013, Three Aspects of Space, http://thedominantmood.wordpress.com
HILL, J., 2005, Drawing Forth, Chapter in Proceedings The Unthinkable Doctorate, Brussels, Sint Lucas, pp.223-227

MASLEN, M., SOUTHERN, J., 2011 , Drawing Projects - An Exploration of the Language of Drawing, London, Black Dog Publishing

MCCALL, C., GRISEWOOD, J., 2011, The Sense of Drawing - Making Sense Through Sensibility (knowing through drawing), in Proceedings Senses and Sensibility in Lisbon, Lisbon, IADE, pp. 167-182

MEDWAY, P., 1996, Virtual and Material Buildings: Construction and Constructivism, in Architecture and Writing, Written communication, vol. 13, no. 4, pp. 473514 quoted in VERMEERSCH, P.W., 2013, Less Vision, More Senses - Towards a More Multisensory Design Approach in Architecture, (PhD Dissertation) Leuven: KU Leuven

MERLEAU PONTY, M., 1948-2002, Causeries, Paris: Editions du Seuil

NICOLAIDES, K., 1990, The Natural Way To Draw, Boston, Houghton Mifflin Company

LATOUR, B., 1986, Visualisation and Cognition - Thinking With Two Hands, in H. Kuklick (editor) Knowledge and Society Studies in the Sociology of Culture Past and Present , Jai Press vol. 6, pp. 1-40

PALLASMAA, J., 2005, The Eyes of the Skin, United Kingdom, John Wiley \& sons Ltd.

PALLASMAA, J., 2009, The Thinking Hand, United Kingdom, John Wiley \& sons Ltd.

TOLLE, E., 1997, De kracht van het NU, Deventer, AnkhHermes

VAN DER LINDEN, S., 2013, www.sofievanderlinden.be

VAN DONGEN, N., ENGBERSEN, H., MEEUWIS, W., MOUS, M. (EDS.), S.D., Programma Tekenen Derdegraads Bevoegdheid, Breda, Pedagogische Akademie Sint Frans

VERMEERSCH, P.W., 2013, Less Vision, More Senses Towards a More Multisensory Design Approach in Architecture, (PhD Dissertation) Leuven: KU Leuven

VOORTHUIS, J.C.T., 2012, Het Ontwerpgesprek - een Filosofie van het Ontwerpen, Rotterdam: NAI010

Author(s):

Robin Schaeverbeke,

KU Leuven, Faculty of Architecture,

Brussels/Ghent, Belgium

Email: robin.schaeverbeke@luca-arts.be

Hélène Aarts,

TU Eindhoven, Faculty of The Built Environment, The Netherlands

Email: h.m.t.aarts@bwk.tue.nl

Ann Heylighen,

KU Leuven, Dept. of Architecture, Research[x]Design, Leuven, Belgium

Email: ann.heylighen@asro.kuleuven.be 